\title{
Analysis of the nutritional priorities, practices and preferences of athletes and active individuals to inform new food product development in sports nutrition
}

\author{
C.C. Carey ${ }^{1}$, L. Doyle ${ }^{2}$ and A. Lucey ${ }^{1}$ \\ ${ }^{1}$ Cork Centre for Vitamin D and Nutrition Research, School of Food and Nutritional Sciences, University College \\ Cork, Cork, Ireland and \\ ${ }^{2}$ Department of Sport and Exercise Science, Waterford Institute of Technology, Waterford, Ireland
}

Sport and exercise nutrition is a rapidly growing sector with increasing demand for evidence-based products to support both competitive and healthy lifestyles. Transitions towards personalised ${ }^{(1)}$ and periodised ${ }^{(2)}$ nutrition practices for sport have led to considerable improvements in exercise performance and training adaptation. The aim of this research is to gain an understanding of the practices, perceived priorities and product preferences of athletes and active individuals, this data would provide important insight for the development of new scientifically informed food products to support performance nutrition.

A questionnaire assessing nutritional practice, perceived priorities and product preferences was devised and received university ethical approval. Questionnaire design was informed and piloted by athletes and active individuals over a series of focus-group sessions. Participants were $\geq 18$ years and actively participated in competitive sport or structured physical activity (PA) ( $\geq 2$-sessions/week). Data collection included multiple-choice, rating scale, rank order and matrix scale questions, which was distributed using Qualtrics (March-May 2020). Data was analysed using IBM SPSS and is presented as descriptive statistics.

A total of 405 (164f, 241m) participants aged $29 \pm 9$ (mean \pm SD) years were included in the final analysis. Of these, $73 \%$ of participants reported active participation in competitive sport while $27 \%$ participants undertook non-competitive structured PA exclusively. Participants performed 10.3 \pm 6.6 hours per week of PA. When asked to rank their top three most desired effects of a sports nutrition product; enhanced muscular recovery was most frequently prioritised receiving 101 first choice preferences and 295 top 3 preferences. Of all participants, 58\% reported regular use of nutritional supplements, with $51 \%$ of all participants noting use for health and wellbeing purposes and $35 \%$ reporting use for exercise performance or recovery. When presented with a list of functional foods; Caffeine-containing functional foods were the most commonly consumed, with $33 \%$ reporting use in relation to exercise performance. When asked to rank the importance of various features of a sports nutrition food product, nutritional profile ranked as most important (3.4 \pm 0.7 out of 4$)$; followed by taste $(3.2 \pm 0.8)$ and accessibility $(3.1 \pm 0.7)$. Wholefood type products received the most first preference selections and most top 3 selections when presented with a number of popular performance and recovery products on the market.

The global movement towards food first approaches for sport nutrition ${ }^{(3,4)}$ provides a unique opportunity for the development of evidence-based functional foods. This lead and end-user validated survey indicates that the availability of evidence-based foods is well received and expected by the sports nutrition sector. Nutritional profile, taste, and accessibility rank as key consumer priorities to inform new product development with whole food product types the most desirable. Foods to support muscle recovery are of particular value to users and the fundamental science to underpin merits further research.

\section{References}

1. Ordovas JM, Ferguson LR, Tai ES, et al. (2018) Brit Med J

2. Stellingwerff T, Morton JP \& Burke LM (2019) Int J Sport Nutr Exerc Metab

3. Sport Ireland Anti Doping Supplements Policy (2017)

4. WADA (2021) World Anti-Doping Code 